\title{
A City Traffic library
}

\author{
Eashan Liyana $^{1} \quad$ Simon Lacroux $^{1} \quad$ Jean-Baptiste Barbe $^{1}$ \\ ${ }^{1}$ Digital Product Simulation, \\ La Celle-Saint-Cloud, France, \\ eashan.liyanaedps-fr.com simon.lacroux@dps-fr.com jean-baptiste.barbe@dps-fr.com
}

\begin{abstract}
Digital Product Simulation (DPS) created a library for the modeling of city traffic. This library is designed for the development and evaluation of control strategies, rendered possible when vehicles are able to communicate between each other and with their infrastructure. CityTraffic library allows for the implementation of control strategies by all of the players acting in an urban environment (e.g. located in vehicles, with a global server computing set points for the vehicles, or with a traffic management system setting speed limits and traffic light cycles).

The library is divided in two parts, macroscopic traffic and microscopic traffic. Macroscopic components are used to describe road networks such as highways whereas microscopic components allow for modeling city traffic where interactions between vehicles and their environment are many.

By using a City Traffic library, cities can decrease the number of traffic jams on their road network, and improve the overall impact of the traffic on the environment.
\end{abstract}

Keywords: Macroscopic and microscopic road sections, City Traffic, Intersections, Navigation models, Map Creator, Vehicles with their global consumption

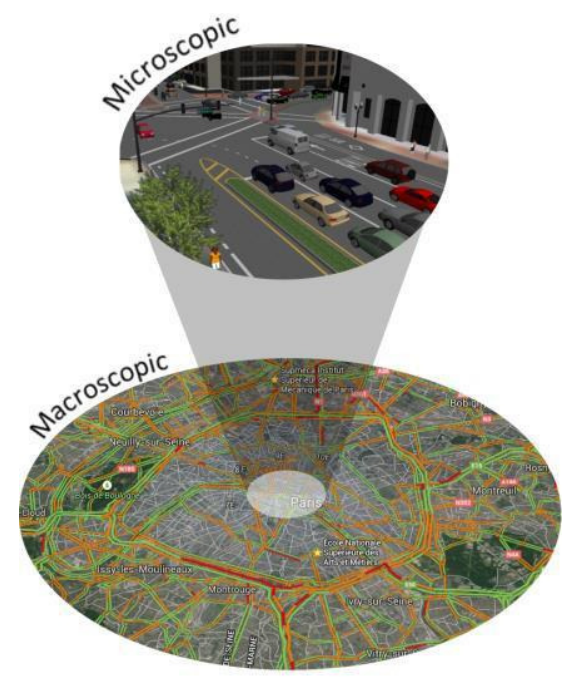

Figure 1: Microscopic and Macroscopic scale

\section{Introduction}

The management of city traffic and the reduction of motor vehicle emissions are more newsworthy than ever.

As part of the MODRIO project (MOdel DRIven physical systems Operation), a European project financed by the ITEA2 program and lead by EDF, DPS was in charge of the development of a Modeling and Simulation CityTraffic library for a city with two different scales, in order to observe both the flow of vehicles on the road, and the interactions between vehicles and their environment (other vehicles and urban infrastructures).

\section{Presentation of the CityTraffic library}

The CityTraffic library is divided in two main packages which are the Microscopic scale and the Macroscopic scale. A connection between those scales was implemented as well.

\subsection{Macroscopic environment}

This modeling scale encompasses an overview of the urban traffic, considering the vehicle flow rate (number of vehicles per hour), not individual vehicles movements. It is based on an analogy with Hydraulics where roads behave as pipes, where intersections are loads, and where vehicles are represented as a fluid.
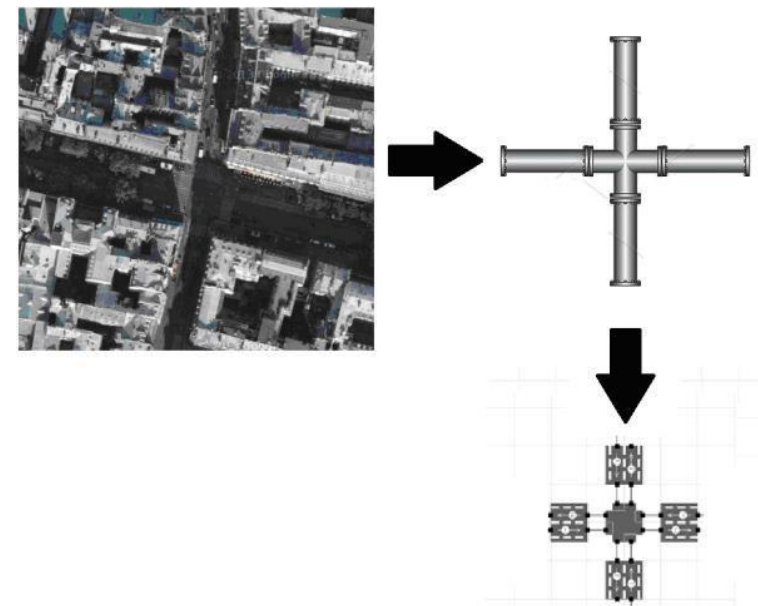

Figure 2: Hydraulic analogy of the macroscopic scale 
The macroscopic models are based on the following variables:

- The density of the traffic : $\rho(v e ́ h / k m)$

- The velocity of vehicles : $v(\mathrm{~km} / \mathrm{h})$

- The vehicle flow rate : $Q(v e ́ h / h)=\rho \cdot v$

The fundamental equation of the macroscopic environment computes the number of vehicles (Aw \& Rascle, 2010) (Iordanova, 2006) (Papageorgiou, 2003)

$$
\frac{\partial \rho}{\partial t}+\frac{\partial(\rho \cdot v)}{\partial x}=0
$$

Several models were implemented around those variables (first and second order models) with different laws applied to the velocity of the vehicles.

Those models were integrated into components to model roads, intersections or sources of vehicles. The following figure is an example of the components for a road section with two lanes of traffic and two sources of traffic flow thus specifying the number of vehicles generated for the section.

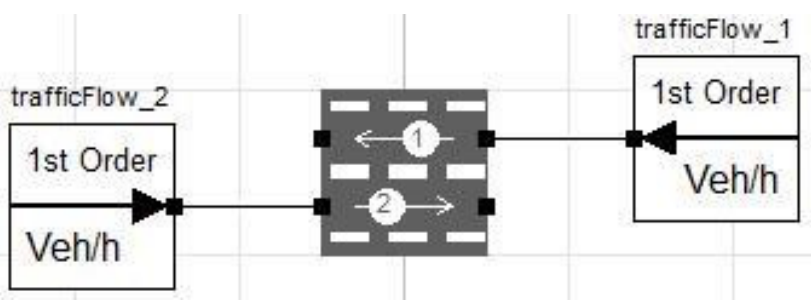

Figure 3: Traffic flow sources for a macroscopic road

Each road is given a maximum density parameter representing the capacity of the road (maximum number of vehicles on the section at the same time). This parameter allows for simulating congestions, that is when the density is higher than this predefined value.

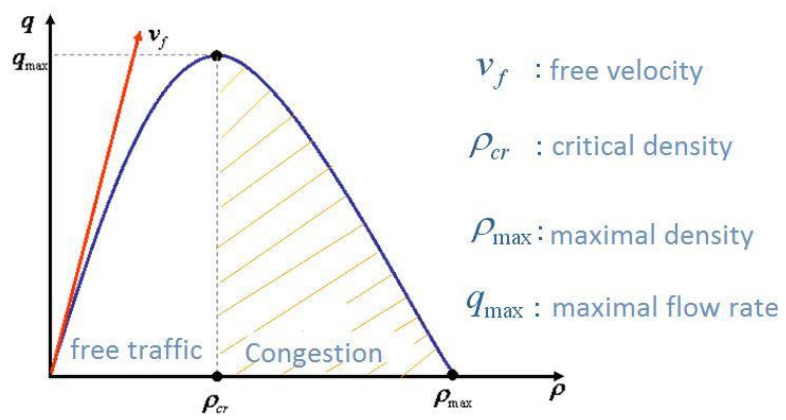

Figure 4: Flow rate as a function of the density of vehicle

This scale gives a really short simulation time, but simulation of interactions between vehicles is impossible. Nevertheless, the macroscopic environment is well suited to modelling highways for which there is no intersection and where the traffic is more fluid than in cities.

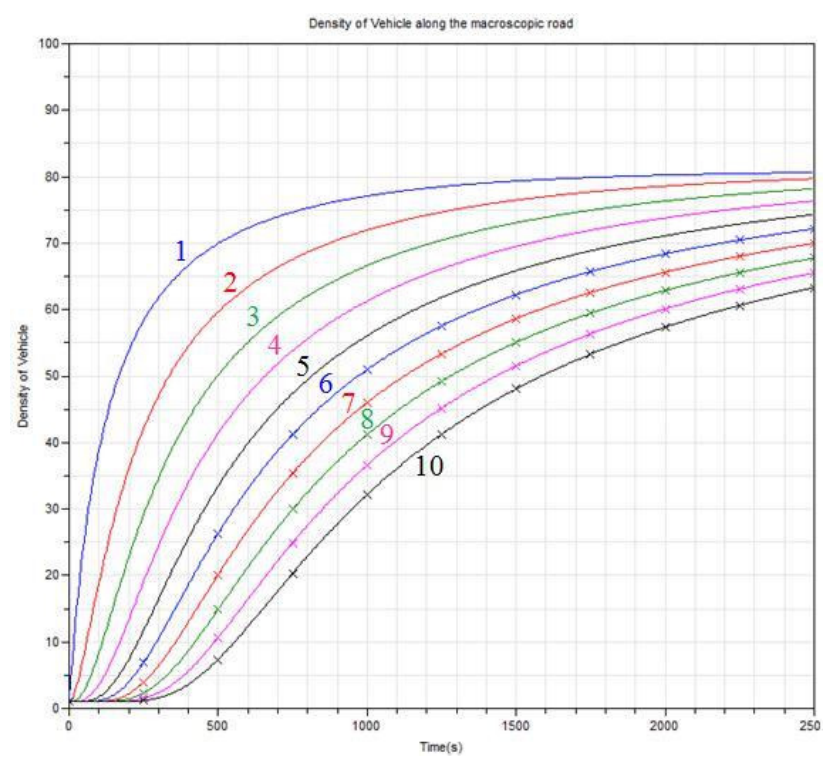

Figure 5: Vehicle density evolution in a $10 \mathrm{~km}$ length road over 10 sections of $1 \mathrm{~km}$

\subsection{Microscopic Environment}

This other scale is based on an insider point of view of the urban traffic. It allows for visualizing the journey of each vehicle and modeling the interactions between vehicles as well as between the vehicles and the urban infrastructures (crossroad, red light, stop sign...).
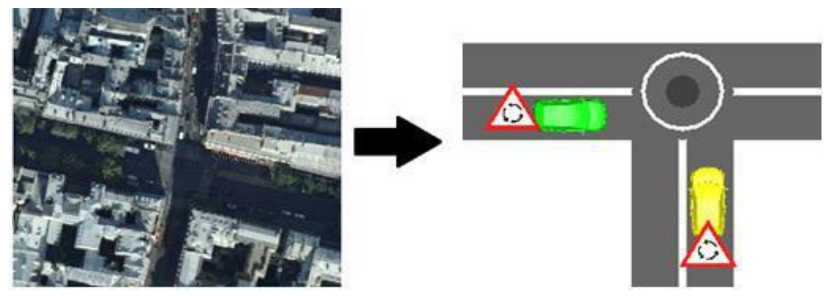

Figure 6: Microscopic scale of a roundabout intersection

The microscopic environment is composed of a Map with a first model named "MapDesign", which is used by a second model named "Env_Micro_and_macro", where the user can define different types of crossroads and initialize the vehicles.

The vehicle model contains a navigation function and a velocity model which are described in part 3.2 of this document. A fuel consumption and a $\mathrm{CO} 2$ emission models were added in order to compute the environmental impact of each vehicle.

\subsection{Connection between environments}

The CityTraffic library includes the possibility to connect the macroscopic and microscopic environments. The simulation time may be reduced by modeling highways or big roads without intersections in the macroscopic scale. 


\subsection{Performance comparison}

In the previous sections we introduced two ways of modeling traffic flow, macroscopic and microscopic. The following figure compares the performance of the two methods.

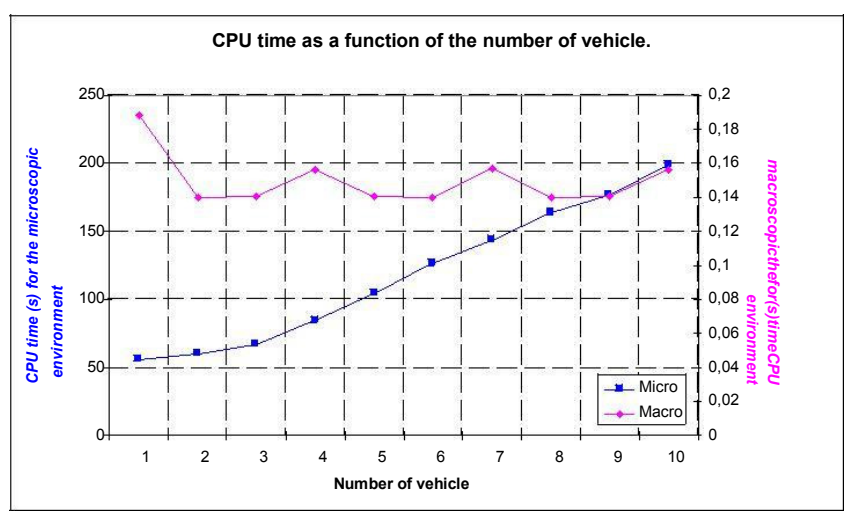

Figure 7: Simulation time as function of the number of vehicles

We simulated a $100 \mathrm{~m}$ length road for different numbers of vehicle. For one car, the macroscopic model is 300 times faster than the microscopic model, and for 10 cars it's one thousand times faster.

Using the macroscopic method to model areas where interactions are low shortens the simulation time.

\section{Content of the library}

\subsection{Map Generation}

The basis of the microscopic environment is the Map where the vehicles move. As for the macroscopic environment for which there is no animation, the map model and the vehicle model are not used.

The Map is composed of nodes whose Cartesian coordinates $(\mathrm{x}, \mathrm{y}, \mathrm{z})$ are given by the user. Those nodes are then linked with each other, and the lanes of circulation can be defined (one way street or two way traffic). Finally, each road includes a speed limit with a maximum velocity to be observed by the vehicles.

Data of the Map is stored in a spreadsheet (CSV file), which is a matrix with the coordinates of the nodes on the diagonal, and in the other locations the maximum velocity in $\mathrm{m} / \mathrm{s}$ for the section between two nodes. In the Map figure 6 , nodes $2(0,100,0)$ and 1 $(0,0,0)$ are set in both directions with a speed limit of $50 \mathrm{~km} / \mathrm{h}(13.88 \mathrm{~m} / \mathrm{s})$. The connections between nodes are modelled with the value of the velocity: between node 1 and node 2 , the velocity is in the first row and second column; the second row and first column corresponding with the velocity between node 2 and node 1 .

On the contrary, nodes $3(0,200,0)$ and $1(0,0,0)$ are not connected, and the value for the maximum velocity between them is $0 \mathrm{~m} / \mathrm{s}$.

If a road is a one way street, the velocity between nodes will be equal to the maximum velocity for the right way of traffic and will be set at $0 \mathrm{~m} / \mathrm{s}$ for the other way. For example, between node 5 and node 4 , the traffic goes from $4=>5$ (in the row of node 4 , there is a value of $13.88 \mathrm{~m} / \mathrm{s}$ in the column of node 5), but from 5 $\Rightarrow 4$ is the wrong way and in the row of node 5 there is $0 \mathrm{~m} / \mathrm{s}$ in the column of node 4 (in red in the figure).

In addition to the CSV file generation, the MapDesign model also creates an animation of the Map where each section is numbered. Roads with a double way of circulation are larger than one way streets, and have two numbers (one for each way of circulation). When there are two numbers, the smaller represents the circulation from the node with the smallest number to the node with the biggest number.

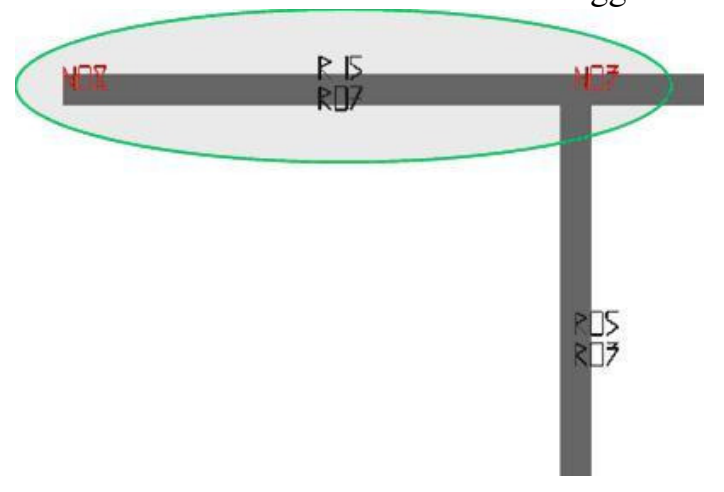

Figure 8: Numbering of the Map sections

For example, on figure 8 , section $\mathrm{R} 7$ is the road between nodes 3 and 8 for the direction N3 $\Rightarrow$ N8, whereas R15 corresponds to the direction N8 $=>$ N3. 


\begin{tabular}{|c|c|c|c|c|c|c|c|c|}
\hline $0,0,0$ & 13.88 & 0 & 0 & 0 & 0 & 0 & 0 & 0 \\
\hline $13: 8 \$$ & $0,100,0$ & 1388 & 0 & 1388 & 0 & 0 & 0 & 0 \\
\hline 0 & 1388 & 0,$200 ; 0$ & 0 & 0 & 13.88 & 0 & $13: s s$ & 0 \\
\hline 0 & 0 & 0 & $100,0,0$ & 1388 & 0 & 0 & 0 & 0 \\
\hline 0 & 13.88 & 0 & 0 & $100,100,0$ & 13.88 & $13.8 \mathrm{~s}$ & 0 & 10 \\
\hline 0 & 0 & 1388 & 0 & 0 & $100,200,0$ & 0 & 0 & $13: 88$ \\
\hline 0 & 0 & 0 & 0 & 13.88 & 0 & $200,100,0$ & 0 & 0 \\
\hline 0 & 0. & 1388 & 0 & 0 & 0 & 0 & $-100 ; 200 ; 0$ & 0 \\
\hline 0 & 0 & 0. & 0 & 0 & 13.88 & 0 & 0 & $200,200,0$ \\
\hline
\end{tabular}

Figure 9: CSV File of a Map with 9 nodes

\subsection{Vehicle model}

\subsubsection{Velocity model}

Three velocity models were created in the CityTraffic library. The first one is named "Simple" and does not take into account the other vehicles. It defines only a constant velocity. The other two models (Krauss and Gipps) are car-following models (Krauß, 1998), meaning the velocity of the selected vehicle is associated with the velocity of the vehicle ahead. If there is no vehicle ahead, the velocity of the vehicle is linked with the velocity of a virtual vehicle located far away, and it accelerates until the velocity reaches the maximum value allowed on the current road section.

Unfortunately, the Krauss model is not realistic, because acceleration and deceleration are instant instead of being gradual. Finally a better model was created: the Gipps model.

This model is used in many traffic modeling software, and includes a progressive acceleration and deceleration with programmable maximum values. Nevertheless, Gipps corresponds to a perfect driver who respects the Highway Code and the security distance between vehicles. So it does not permit to model an overtaking because the velocity of the vehicle will always be inferior or equal to the velocity of the vehicle ahead. Future development will solve this issue.

For roads with two ways of circulation, a function was created to identify vehicles coming from the opposite way. Then, a passing vehicle will not make the vehicle brake, because it will not consider it as a vehicle ahead.

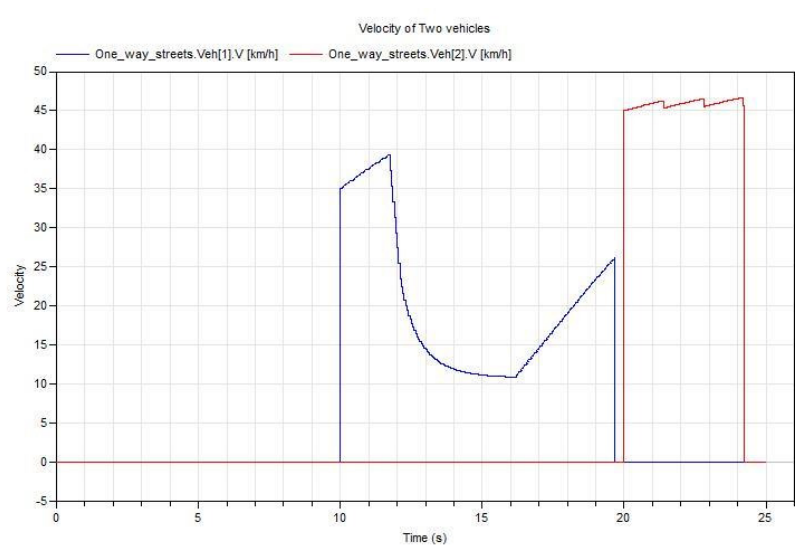

Figure 10: Simulation of two vehicles. The blue car decelerates to turn then accelerates until reaching its destination. The red car is on a straight way and its velocity is limited by the road's speed limit.

\subsubsection{Navigation function}

The navigation function of the vehicle is based on the "Dijkstra algorithm" (Dijkstra, 1959) which identifies nodes to cross, in order to reach the destination using the shortest way in terms of distance. This algorithm uses the following methodology:

From one node, the function will first determine the nodes which are connected to it.

If a node is not connected to the first one, its "cost" will be set to the infinite value, whereas the connected nodes will have a cost proportional to the distance between the nodes. Then the same process is repeated for all the nodes of the Map until the destination node is reached.

Finally, the nodes are classified by their cost, and the table of nodes returned as output will be a table of the nodes with a minimal cost. 


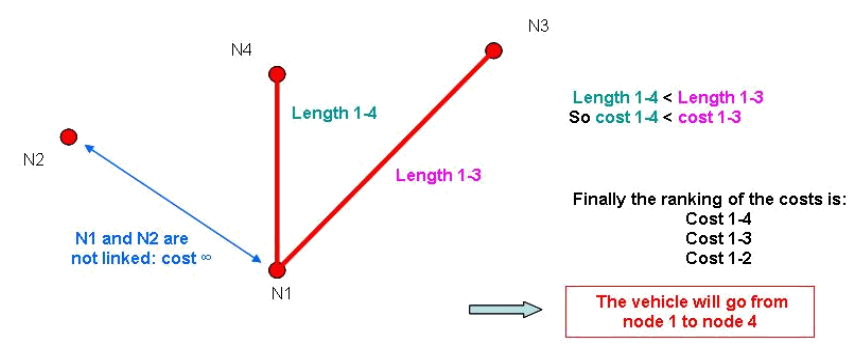

Figure 11: Principle of the Dijkstra algorithm

\subsubsection{Types of vehicle}

In order to be able to visualize the vehicles on the Map, CAD models are imported for each type of vehicle.

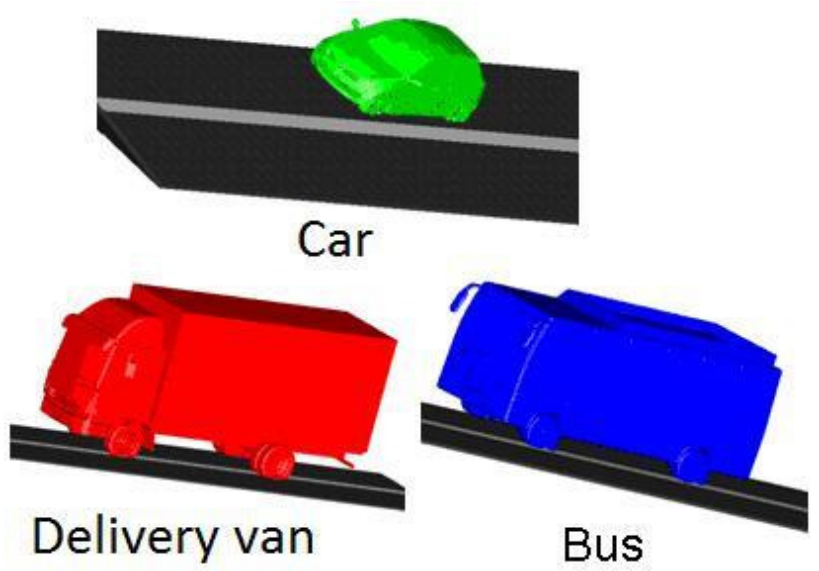

Figure 12: Types of vehicles available

Each type of vehicle has its own characteristics (dimensions, mass...) which are used to update the safety distance between vehicles and to compute the fuel consumption of each vehicle.

\subsubsection{Fuel consumption and $\mathrm{CO}_{2}$ emissions}

The fuel consumption model is based on the computing of the traction force (Guzzella \& Sciarretta, 2007) (Eriksson, 2013) (SETRA, 2009). In order for the velocity and the acceleration to be constant during a short interval of time, a time stamp is used when computing all of the variables. Thus the traction power is evaluated using a quasi-static method.

With the power calculated, the fuel consumed power "Pf" is computed using the efficiencies of the elements of the transmission.

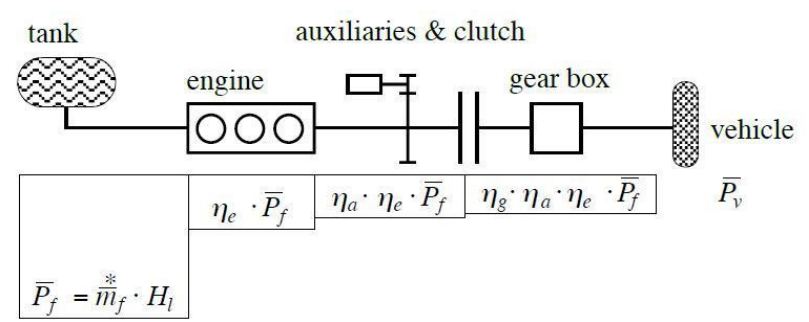

Figure 13: Transmission chain of a vehicle

The engine efficiency is the most difficult to compute, because it depends on the rotational speed of the engine and the ratio of the gear box.
The equation for the flow of fuel is:

$$
V_{f}^{*}=\bar{P}_{f} /\left(H_{l} \cdot \rho_{f}\right)
$$

With for the diesel:

- $\mathrm{Hl}=43.5 * 10^{\wedge} 6 \mathrm{~J} / \mathrm{kg}$ for the fuel's lower heating value

- $\rho f=0.75 \mathrm{~kg} / \mathrm{L}$ for the density.

$\mathrm{CO} 2$ emissions are calculated with the following equation:

$\mathrm{CO} 2$ emission $=$ Fuel consumption $* \rho \mathrm{f} * \mathrm{Hl} *$

fuel emission factor $*$ partial combustion factor

These models are included in the vehicle model to determine the exact quantity of fuel consumed and $\mathrm{CO} 2$ emitted during the trip of the vehicle on the Map.

\section{Intersection models}

Five models of intersection were created (roundabout, traffic light, stop sign, yield and right of way), and three models of stops (bus station, delivery area and pedestrian crossing). Those models can be activated only for the microscopic environment via an interface where the user defines locations of the intersections and stops on the Map by specifying nodes and sections of the intersection.

\subsection{Ghost vehicles}

When a vehicle "A" reaches an intersection, a "ghost vehicle" is set in its place with a $0 \mathrm{~m} / \mathrm{s}$ velocity. These ghost vehicles are present on the map but not shown on the animation by Dymola.

As the velocity model detects the presence of the vehicle ahead, the vehicle "A" will adapt its velocity to the ghost vehicle and will stop. When the intersection model has checked that all conditions are fulfilled to let the vehicle go forward, the ghost vehicle disappears and the vehicle "A" may leave the intersection.

Figure 14 is an example of this situation at an intersection. There is a yield at node 2. The road section impacted by the yield is the blue one.

The yellow vehicle has to give way to the green vehicles coming from its left and its right, so the intersection model will identify the black road sections as having priority, and a ghost vehicle will appear in front of the yellow vehicle (in pink on the previous figure). The position of this ghost vehicle will be fixed, and its velocity equal to $0 \mathrm{~m} / \mathrm{s}$, so the yellow vehicle will detect it and will stop just behind it.

When green vehicles have passed, the intersection model detects that there is no more vehicles on the priority roads and the ghost vehicle is removed. As the yellow vehicle has no more vehicles ahead, it resumes its trip. 


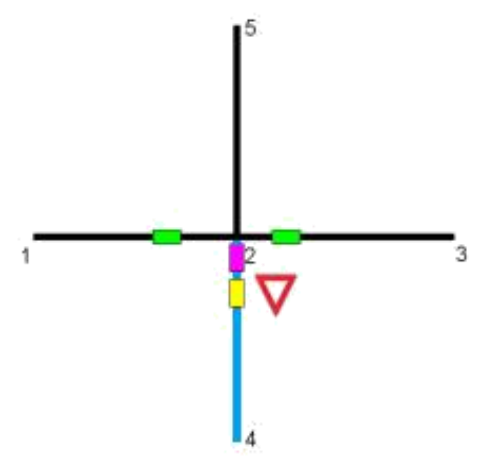

Figure 14: Example of a yield

When the green vehicles have passed, the intersection model detects that there is no more vehicles on the priority roads and the ghost vehicle is removed. As the yellow vehicle has no more vehicles ahead, it resumes its trip.

The following figure shows the animation of an intersection.

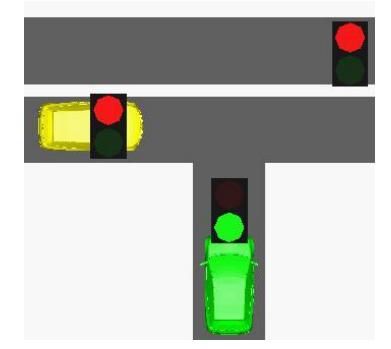

Figure 15: Animation of a red light

\subsection{Stops}

Other models were implemented to stop the vehicles which are not located at the intersection but in the middle of the road. Those models are the bus stations, the delivery places and the pedestrian crossings.

The first two models impact only one type of vehicle (bus or delivery vans), but the pedestrian crossing model stops all vehicles when pedestrians are crossing.

\section{Conclusion}

We presented the CityTraffic library which offers an intuitive tool for modeling interactions between vehicles and their environment. The macroscopic and microscopic scales speed up the simulation while computing the impact of an intersection on the traffic flow and the fuel consumption of a vehicle during its trip.

Other intersection models are being created, as well as a more realistic model for the driver with heterogeneous behaviors.

\section{Acknowledgements}

The French Ministry DGE has funded this work within the ITEA2 project MODRIO.

\section{References}

A. Aw and M. Rascle (2010): Resurrection of "second order" models of traffic flow.

V. Iordanova (2006): Contribution à la modélisation et la commande du trafic routier : Approches par Bond Graph et commande par platitude.

M.Papageorgiou (2003): Review of Road Traffic Control Strategies.

Stefan Krauß (1998): Microscopic Modeling of Traffic Flow:Investigation of Collision Free Vehicle Dynamics.

http://licence-math.univ-

lyon1.fr/lib/exe/fetch.php?media=gla:dijkstra.pdf

Lino Guzzella and Antonio Sciarretta (2007): Vehicle Propulsion Systems, Introduction to Modeling and Optimization.

Lars Eriksson, Linköping University (2013): Vehicle Propulsion Systems, Course Introduction \& Energy System Overview.

SETRA (service d'étude sur les transports, les routes et leurs aménagements) (2009): Etude des émissions routières de polluants atmosphériques. 